\title{
Electrolytes at spherical dielectric interfaces
}

\author{
R. A. Curtis and L. Lue ${ }^{a)}$ \\ School of Chemical Engineering and Analytical Science, The University of Manchester, P.O. Box 88, \\ Sackville Street, Manchester M60 1QD, United Kingdom
}

(Received 22 July 2005; accepted 6 September 2005; published online 31 October 2005)

\begin{abstract}
A variational theory is developed and applied to study the properties of dielectric spheres immersed in a symmetric electrolyte solution. In the limit that the radius of the sphere becomes much larger than the Debye screening length, the system reduces to that of a planar dielectric interface. For this case, the excess surface tension obtained by the variational theory reduces to the Onsager-Samaras [J. Chem. Phys. 2, 528 (1934)] limiting law at low electrolyte concentrations. As the radius of the dielectric sphere decreases, the excess surface tension also decreases. The implications of this work to protein-salt interactions and the salting out of proteins are discussed. (C) 2005 American Institute of Physics. [DOI: 10.1063/1.2102890]
\end{abstract}

\section{INTRODUCTION}

Most biological systems of interest are aqueous electrolyte solutions containing macromolecules or macromolecular structures (e.g., proteins, polysaccharides, micelles, bilayers, etc.). Understanding these systems requires determining the interactions between these colloids and the surrounding aqueous electrolyte solvent and also the solvent-mediated interactions between the colloids. The traditional approach to describing the interaction of the electrolyte solution with the colloid is based on the Poisson-Boltzmann equation. However, one of the inadequacies of these approaches is that they predict an excess of salt in the domain of a charged colloid, whereas for most proteins in moderately concentrated salt solutions, salt is preferentially excluded. ${ }^{1}$ As discussed below, one route to correcting this shortcoming is to include the influence of the low dielectric interior of the colloids (e.g., micelles and proteins) in the models.

Repulsive image charge forces between ions and a low dielectric boundary result in preferential desorption of salt from the interface. By using a Poisson-Boltzmann approach to quantify the desorption, Wagner ${ }^{2}$ and Onsager and Samaras ${ }^{3}$ were able to compute the excess surface tension of electrolyte solutions by integrating the Gibbs adsorption equation. Similarly, studies of protein solutions have shown that the exclusion of salt around the protein is correlated with the surface tension increment of the salt indicating that as a first approximation the interior of a protein could be treated as a low dielectric continuum, similar to air. ${ }^{1}$

The effect of image charges on surface tension and on the force between surfaces is well known for planar geometries (for instance, see Refs. 4-11). However, the influence of curvature on these interactions is less well known. Linse ${ }^{12}$ and Messina ${ }^{13}$ used Monte Carlo simulations to study the effect of image forces on the electrical double layer about a charged colloid sphere with a different dielectric constant from the surrounding medium. In these studies, the density distribution about the spheres is determined, but the resulting

${ }^{a)}$ Electronic mail: leo.lue@manchester.ac.uk surface tension was not calculated. Groenewold extended the work of Onsager and Samaras to weakly curved systems ${ }^{14}$ and found that the surface tension decreased if the surface curved away from the electrolyte solution and increased if it curved toward the solution.

In this work, we examine the excess surface tension of dielectric particles. In particular, we consider a sphere of radius $R$ and dielectric constant $\epsilon^{\prime}$ that is immersed in a continuous medium of dielectric constant $\epsilon$ containing dissolved electrolytes. To study this problem, we pursue a field theoretic formulation of the electrolyte problem. ${ }^{15-18}$ This type of approach has already been applied to a wide range of problems, including electrolytes near planar dielectric interfaces ${ }^{19-21}$ or the potential of mean force between spherical macroions. ${ }^{16,22}$ The advantage of this approach over the traditional Poisson-Boltzmann equation (such as used by Onsager and Samaras and applied by Groenwold to curved dielectric interfaces) is that it is able to account for ion-ion correlation effects and the influence of image charge interactions can be self-consistently incorporated. In addition, other nonelectrostatic interactions experienced by the ions can be included in the model, such as excluded volume forces ${ }^{11}$ or ion-ion and ion-surface dispersion forces. The latter forces are believed to be important in understanding the specific ion effects, which are significant in moderately concentrated salt solutions. $^{23}$

The remainder of the paper is organized as follows. In the Sec. II, we present a brief review of the functional integral formulation of electrolyte systems and apply a standard variational approximation to develop the theory. The application of this theory to bulk electrolyte solutions is given in Sec. III, and its application to an electrolyte at a general dielectric interface is discussed in Sec. IV. The particular examples of planar (where the theory exactly reproduces the Onsager-Samaras limiting law at low electrolyte concentrations) and spherical interfaces are examined in Sec. V and VI, respectively. 


\section{THEORY}

\section{A. Development of the free-energy functional}

In this section, we develop a functional integral formulation of the grand partition function of an electrolyte solution. This type of formulation has already been presented by previous authors, ${ }^{15-18,24}$ so we only present a brief overview of the main points.

Consider an electrolyte system composed of particles with embedded point charges that are immersed in a continuous medium with a spatially varying dielectric constant $\epsilon(\mathbf{r})$ and with a fixed charge distribution $\Sigma(\mathbf{r})$ imposed on the system ( $\mathbf{r}$ denotes the position in the system). The chemical potential of particles of type $\alpha$ is held fixed at $\mu_{\alpha}$, and there is a nonuniform external potential $u_{\alpha}(\mathbf{r})$ acting on each particle. The grand partition function $Z_{G}$ for this system is given by $^{25}$

$$
\begin{aligned}
Z_{G}[\gamma, \Sigma]= & \sum_{N_{1}=0}^{\infty} \cdots \sum_{N_{M}=0}^{\infty} \prod_{\nu} \frac{1}{N_{\nu} ! \lambda_{\nu}^{3 N_{\nu}}} \\
& \times \int \prod_{\pi} d \mathbf{r}_{\pi t} e^{-\beta E^{\text {elec }}-\beta E^{\mathrm{ref}_{+}}+\gamma_{\alpha, \kappa} \gamma_{\alpha}\left(\mathbf{r}_{\alpha, \kappa}\right)},
\end{aligned}
$$

where $\beta=1 /\left(k_{B} T\right), k_{B}$ is the Boltzmann constant, $T$ is the absolute temperature, $N_{\alpha}$ is the number of particles of type $\alpha, \mathbf{r}_{\alpha, \kappa}$ is the position of the $\kappa$ th ion of type $\alpha, \lambda_{\alpha}$ is the thermal wavelength of an ion of type $\alpha$, and $\gamma_{\alpha}(\mathbf{r})=\beta\left[\mu_{\alpha}\right.$ $\left.-u_{\alpha}(\mathbf{r})\right]$. The interaction energy between the particles is divided into an electrostatic contribution $E^{\text {elec }}$ and nonelectrostatic contributions $E^{\text {ref }}$ (e.g., due to excluded volume, etc.).

The energy of the electrostatic field can be written in terms of the instantaneous charge distribution $Q(\mathbf{r}),{ }^{26}$

$$
E^{\mathrm{elec}}=\frac{1}{2} \int d \mathbf{r} d \mathbf{r}^{\prime} Q(\mathbf{r}) G_{0}\left(\mathbf{r}, \mathbf{r}^{\prime}\right) Q\left(\mathbf{r}^{\prime}\right)-\sum_{\alpha, \kappa} e_{\alpha}^{\mathrm{se}}\left(\mathbf{r}_{\alpha, \kappa}\right),
$$

where $G_{0}\left(\mathbf{r}, \mathbf{r}^{\prime}\right)$ is the Green's function associated with the electrostatics problem, which is defined by the following relation:

$$
-\frac{1}{4 \pi} \nabla \cdot \epsilon(\mathbf{r}) \nabla G_{0}\left(\mathbf{r}, \mathbf{r}^{\prime}\right)=\delta\left(\mathbf{r}-\mathbf{r}^{\prime}\right) .
$$

Physically, the Green's function $G_{0}\left(\mathbf{r}, \mathbf{r}^{\prime}\right)$ is the electric potential at position $\mathbf{r}$ that is generated by a unit point charge located at position $\mathbf{r}^{\prime}$.

The first term on the left side of Eq. (2) gives the total electrostatic energy, which includes a contribution from the direct interaction of each point charge with itself. This infinite self-energy is removed by including the second term on the right side of Eq. (2), which gives the self-energy $e_{\alpha}^{\mathrm{se}}(\mathbf{r})$ $=q_{\alpha}^{2} G_{0}^{\text {free }}(\mathbf{r}, \mathbf{r}) / 2$ of a point charge in an infinite medium with a constant dielectric constant $\epsilon$. The Green's function $G_{0}^{\text {free }}$ is given by

$$
G_{0}^{\mathrm{free}}\left(\mathbf{r}, \mathbf{r}^{\prime}\right)=\frac{1}{\epsilon\left|\mathbf{r}-\mathbf{r}^{\prime}\right|} .
$$

The total charge density is composed of a fixed charge distribution $\Sigma(\mathbf{r})$ and a contribution from the mobile particles with embedded point charges:

$$
Q(\mathbf{r})=\sum_{\alpha, \kappa} q_{\alpha} \delta\left(\mathbf{r}-\mathbf{r}_{\alpha, \kappa}\right)+\Sigma(\mathbf{r}),
$$

where $q_{\alpha}$ is the charge of a particle of type $\alpha$.

With this expression for the electrostatic energy, the grand partition function can be rewritten as a functional integral by introducing the Hubbard-Stratonovich transformation $^{27,28}$ to give

$$
\begin{aligned}
Z_{G}[\gamma, \Sigma]= & \frac{1}{\mathcal{N}_{0}} \int \mathcal{D} \psi(\cdot) \\
& \times \exp \left\{-\frac{1}{2 \beta} \int d \mathbf{r} d \mathbf{r}^{\prime} \psi(\mathbf{r}) G_{0}^{-1}\left(\mathbf{r}, \mathbf{r}^{\prime}\right) \psi\left(\mathbf{r}^{\prime}\right)\right. \\
& \left.-\int d \mathbf{r} \Sigma(\mathbf{r}) i \psi(\mathbf{r})+\ln Z_{G}^{\mathrm{ref}}\left[\gamma-q i \psi+\beta e^{\mathrm{se}}\right]\right\},
\end{aligned}
$$

where $Z_{G}^{\mathrm{ref}}$ is the grand partition function of the reference system (i.e., the system with no electrostatic interactions) and

$$
\mathcal{N}_{0}=\int \mathcal{D} \psi(\cdot) \exp \left[-\frac{1}{2 \beta} \int d \mathbf{r} d \mathbf{r}^{\prime} \psi(\mathbf{r}) G_{0}^{-1}\left(\mathbf{r}, \mathbf{r}^{\prime}\right) \psi\left(\mathbf{r}^{\prime}\right)\right] .
$$

The function $i \psi(\mathbf{r})$ can be interpreted as being equal to an instantaneous value of the electrostatic potential, and the functional integral can be thought of as an integral over all possible "shapes" of the electrostatic potential due to the thermal motion of the electrolytes.

\section{B. The variational method}

The functional integral representation for the grand potential given in Eq. (5) is exact, although approximate methods are required to solve this equation, such as the meanfield approximation or the loop expansion. In this work, we use a standard variational method, ${ }^{29}$ which has recently been used to study ${ }^{30}$ electrolyte systems near highly charged objects.

Within the variational method, calculations are performed with respect to a reference Hamiltonian, which is typically chosen to be Gaussian. The most general form for a Gaussian Hamiltonian is

$$
\begin{aligned}
-\mathcal{H}_{\mathcal{K}}[\psi]= & \frac{1}{2 \beta} \int d \mathbf{r} d \mathbf{r}^{\prime}[\psi(\mathbf{r})-\bar{\psi}(\mathbf{r})] \\
& \times G_{\mathcal{K}}^{-1}\left(\mathbf{r}, \mathbf{r}^{\prime}\right)\left[\psi\left(\mathbf{r}^{\prime}\right)-\bar{\psi}\left(\mathbf{r}^{\prime}\right)\right],
\end{aligned}
$$

where $\bar{\psi}$ is the mean value of the field $\psi$,

$$
G_{\mathcal{K}}^{-1}\left(\mathbf{r}, \mathbf{r}^{\prime}\right)=G_{0}^{-1}\left(\mathbf{r}, \mathbf{r}^{\prime}\right)+\mathcal{K}\left(\mathbf{r}, \mathbf{r}^{\prime}\right),
$$

and $\mathcal{K}$ is an arbitrary screening function. The modified Green's function $G_{\mathcal{K}}\left(\mathbf{r}, \mathbf{r}^{\prime}\right)$ represents the electric potential at position $\mathbf{r}$ generated by a unit point charge located at position $\mathbf{r}^{\prime}$. It differs from the "bare" Green's function $G_{0}\left(\mathbf{r}, \mathbf{r}^{\prime}\right)$ because it includes the presence of other charged particles in the system through the screening function $\mathcal{K}$. 
Averages taken with respect to the Gaussian Hamiltonian, given in Eq. (6), are denoted by $\langle(\cdots)\rangle_{\mathcal{K}}$ and are defined as

$$
\begin{aligned}
\langle(\cdots)\rangle_{\mathcal{K}} \equiv & \frac{1}{\mathcal{N}_{\mathcal{K}}} \int \mathcal{D} \delta \psi(\cdot)(\cdots) \exp \left[-\frac{1}{2 \beta}\right. \\
& \left.\times \int d \mathbf{r} d \mathbf{r}^{\prime} \delta \psi(\mathbf{r}) G_{\mathcal{K}}^{-1}\left(\mathbf{r}, \mathbf{r}^{\prime}\right) \delta \psi\left(\mathbf{r}^{\prime}\right)\right],
\end{aligned}
$$

where $\delta \psi(\mathbf{r})=\psi(\mathbf{r})-\bar{\psi}(\mathbf{r})$, and the normalization constant $\mathcal{N}_{\mathcal{K}}$ is given by

$$
\mathcal{N}_{\mathcal{K}}=\int \mathcal{D} \psi(\cdot) \exp \left[-\frac{1}{2 \beta} \int d \mathbf{r} d \mathbf{r}^{\prime} \psi(\mathbf{r}) G_{\mathcal{K}}^{-1}\left(\mathbf{r}, \mathbf{r}^{\prime}\right) \psi\left(\mathbf{r}^{\prime}\right)\right] .
$$

The grand partition function can then be rewritten in terms of the Gaussian Hamiltonian,

$$
\begin{aligned}
\ln Z_{G}[\gamma, \Sigma]= & \frac{1}{2 \beta} \int d \mathbf{r} d \mathbf{r}^{\prime} i \bar{\psi}(\mathbf{r}) G_{0}^{-1}\left(\mathbf{r}, \mathbf{r}^{\prime}\right) i \bar{\psi}\left(\mathbf{r}^{\prime}\right) \\
& -\int d \mathbf{r} \Sigma(\mathbf{r}) i \bar{\psi}(\mathbf{r})+\ln \frac{\mathcal{N}_{\mathcal{K}}}{\mathcal{N}_{0}}+\ln \left\langle e^{-\delta H_{\mathcal{K}}[\delta \psi \psi}\right\rangle_{\mathcal{K}}
\end{aligned}
$$

where the fluctuation Hamiltonian $\delta H_{\mathcal{K}}$ is given by

$$
\begin{aligned}
-\delta H_{\mathcal{K}}[\delta \psi]= & \ln Z_{G}^{\mathrm{ref}}\left[\gamma-q i \delta \psi-i q \bar{\psi}+\beta e^{\mathrm{se}}\right] \\
& -\frac{1}{2 \beta} \int d \mathbf{r} d \mathbf{r}^{\prime} i \delta \psi(\mathbf{r}) \mathcal{K}\left(\mathbf{r}, \mathbf{r}^{\prime}\right) i \delta \psi\left(\mathbf{r}^{\prime}\right) \\
& -\int d \mathbf{r}\left[\Sigma(\mathbf{r})-\frac{1}{\beta} \int d \mathbf{r}^{\prime} G_{\mathcal{K}}^{-1}\left(\mathbf{r}, \mathbf{r}^{\prime}\right) i \bar{\psi}\left(\mathbf{r}^{\prime}\right)\right] \\
& \times i \delta \psi(\mathbf{r}) .
\end{aligned}
$$

Using the following relation for the Gaussian fluctuation term: ${ }^{20}$

$$
\begin{aligned}
\ln \frac{\mathcal{N}_{\mathcal{K}}}{\mathcal{N}_{0}} & =-\frac{1}{2} \int_{0}^{1} d \zeta \int d \mathbf{r} d \mathbf{r}^{\prime} \mathcal{K}\left(\mathbf{r}, \mathbf{r}^{\prime}\right) G_{\zeta \mathcal{K}}\left(\mathbf{r}, \mathbf{r}^{\prime}\right) \\
& =-\frac{1}{2} \int_{0}^{1} d \zeta \operatorname{Tr} \mathcal{K} G_{\zeta \mathcal{K}},
\end{aligned}
$$

the final expression for the grand partition function becomes

$$
\begin{aligned}
\ln Z_{G}[\gamma, \Sigma]= & \frac{1}{2 \beta} \int d \mathbf{r} d \mathbf{r}^{\prime} i \bar{\psi}(\mathbf{r}) G_{0}^{-1}\left(\mathbf{r}, \mathbf{r}^{\prime}\right) i \bar{\psi}\left(\mathbf{r}^{\prime}\right) \\
& -\int d \mathbf{r} \Sigma(\mathbf{r}) i \bar{\psi}(\mathbf{r})-\frac{1}{2} \int_{0}^{1} d \zeta \operatorname{Tr} \mathcal{K} G_{\zeta \mathcal{K}} \\
& +\ln \left\langle e^{-\delta H_{\mathcal{K}}[\delta \psi]}\right\rangle_{\mathcal{K}},
\end{aligned}
$$

where $G_{\zeta \mathcal{K}}^{-1}\left(\mathbf{r}, \mathbf{r}^{\prime}\right)=G_{0}^{-1}\left(\mathbf{r}, \mathbf{r}^{\prime}\right)+\zeta \mathcal{K}\left(\mathbf{r}, \mathbf{r}^{\prime}\right)$. Equation (11) is an exact expression for the grand partition function; however, the final term of this expression cannot be evaluated analytically, and, consequently, some method must be used to approximate its value, such as the variational method.
The variational method is based on the observation that the exact grand partition function is independent of the choice of the functions $\bar{\psi}$ and $\mathcal{K}$. Mathematically, this can be expressed as

$$
\begin{aligned}
& \frac{\delta}{\delta i \bar{\psi}(\mathbf{r})} \ln Z_{G}[\gamma, \Sigma]=0, \\
& \frac{\delta}{\delta \mathcal{K}\left(\mathbf{r}, \mathbf{r}^{\prime}\right)} \ln Z_{G}[\gamma, \Sigma]=0 .
\end{aligned}
$$

While the exact grand partition function is completely independent of the form of the functions $\bar{\psi}$ and $\mathcal{K}$, any approximations to the grand partition function will depend on these functions. The idea of the variational method is to make some approximation to the grand partition function and to choose the functions $\bar{\psi}$ and $\mathcal{K}$ such that Eqs. (12) and (13) are satisfied.

A cumulant expansion can be used to approximate the final term in Eq. (11). If this expansion is limited to first order, then the following inequality is obatined (due to the convexity of the exponential function ${ }^{29}$ ):

$$
\begin{aligned}
\ln \left\langle e^{-\delta H_{\mathcal{K}}[\delta \psi]}\right\rangle_{\mathcal{K}} \geqslant & \left\langle\left(-\delta H_{\mathcal{K}}[\delta \psi]\right)\right\rangle_{\mathcal{K}} \\
\geqslant & \left\langle\ln Z_{G}^{\mathrm{ref}}\left[\gamma-q i \psi-q i \bar{\psi}+\beta e^{\mathrm{se}}\right]\right\rangle_{\mathcal{K}} \\
& +\frac{1}{2} \operatorname{Tr} \mathcal{K} G_{\mathcal{K}},
\end{aligned}
$$

which leads to

$$
\begin{aligned}
\ln Z_{G}[\gamma, \Sigma] \geqslant & \frac{1}{2 \beta} \int d \mathbf{r} d \mathbf{r}^{\prime} i \bar{\psi}(\mathbf{r}) G_{0}^{-1}\left(\mathbf{r}, \mathbf{r}^{\prime}\right) i \bar{\psi}\left(\mathbf{r}^{\prime}\right) \\
& -\int d \mathbf{r} \Sigma(\mathbf{r}) i \bar{\psi}(\mathbf{r})-\frac{1}{2} \int_{0}^{1} d \zeta \operatorname{Tr} \mathcal{K}\left(G_{\zeta \mathcal{K}}\right. \\
& \left.-G_{\mathcal{K}}\right)+\left\langle\ln Z_{G}^{\mathrm{ref}}\left[\gamma-q i \psi-q i \bar{\psi}+\beta e^{\mathrm{se}}\right]\right\rangle_{\mathcal{K}}
\end{aligned}
$$

The exact grand partition function has a value that is always greater than the approximation given on the right side of Eq. (15). This approximation depends on the functions $\bar{\psi}$ and $\mathcal{K}$, that, in the variational method, are chosen to satisfy Eqs. (12) and (13). With these choices, the right side of Eq. (15) is also maximized. Note that the variational approach for the grand partition function can be systematically improved by including higher-order cumulants; ${ }^{30,31}$ however, in this case, the inequality no longer applies.

In this work, only electrostatic interactions are considered, in which case $Z_{G}^{\text {ref }}$ corresponds to an ideal-gas mixture. The ideal-gas reference partition function is given by

$$
\ln Z_{G}^{\mathrm{ref}}[\gamma]=\sum_{\alpha} \lambda_{\alpha}^{-d} \int d \mathbf{r} e^{\gamma_{\alpha}(\mathbf{r})}
$$

and the grand partition function becomes 


$$
\begin{aligned}
\ln Z_{G}[\gamma, \Sigma] \geqslant & \frac{1}{2 \beta} \int d \mathbf{r} d \mathbf{r}^{\prime} i \bar{\psi}(\mathbf{r}) G_{0}^{-1}\left(\mathbf{r}, \mathbf{r}^{\prime}\right) i \bar{\psi}\left(\mathbf{r}^{\prime}\right) \\
& -\int d \mathbf{r} \Sigma(\mathbf{r}) i \bar{\psi}(\mathbf{r}) \\
& -\frac{1}{2} \int_{0}^{1} d \zeta \operatorname{Tr} \mathcal{K}\left(G_{\zeta \mathcal{K}}-G_{\mathcal{K}}\right) \\
& +\sum_{\alpha} \lambda_{\alpha}^{-d} \int d \mathbf{r} e^{\gamma_{\alpha}(\mathbf{r})-q_{\alpha} i \bar{\psi}(\mathbf{r})-\left(\beta q_{\alpha}^{2} / 2\right) \Delta G_{\mathcal{K}}(\mathbf{r}, \mathbf{r})}
\end{aligned}
$$

where

$$
\Delta G_{\mathcal{K}}(\mathbf{r}, \mathbf{r}) \equiv G_{\mathcal{K}}(\mathbf{r}, \mathbf{r})-G_{0}^{\mathrm{free}}(\mathbf{r}, \mathbf{r}) .
$$

Here, $\Delta G_{\mathcal{K}}(\mathbf{r}, \mathbf{r})$ is the free energy of transferring an ion from an infinitely dilute solution to an electrolyte solution at finite concentration.

The first variational condition [Eq. (12)] reduces to

$$
-\frac{1}{4 \pi \beta} \nabla \cdot \epsilon(\mathbf{r}) \nabla i \bar{\psi}(\mathbf{r})=\sum_{\alpha} q_{\alpha} \rho_{\alpha}(\mathbf{r})+\Sigma(\mathbf{r}),
$$

where $\rho_{\alpha}(\mathbf{r})$ is the density of species $\alpha$, which can be calculated from the grand partition function according to

$$
\rho_{\alpha}(\mathbf{r}) \equiv \frac{\delta \ln Z_{G}[\gamma, \Sigma]}{\delta \gamma_{\alpha}(\mathbf{r})}=\lambda_{\alpha}^{-d} e^{\gamma_{\alpha}(\mathbf{r})-q_{\alpha} i \bar{\psi}(\mathbf{r})-\left(\beta q_{\alpha}^{2} / 2\right) \Delta G_{\mathcal{K}}(\mathbf{r}, \mathbf{r})}
$$

Equation (19) is the Poisson equation with the electric potential $i \bar{\psi}(\mathbf{r}) / \beta$. The second variational condition [Eq. (13)] leads to an expression for the screening function $\mathcal{K}$ :

$$
\mathcal{K}\left(\mathbf{r}, \mathbf{r}^{\prime}\right)=\delta\left(\mathbf{r}-\mathbf{r}^{\prime}\right) \sum_{\alpha} \beta q_{\alpha}^{2} \rho_{\alpha}(\mathbf{r})
$$

\section{BULK ELECTROLYTE}

In this section, we demonstrate that the variational theory yields the same results as the Debye-Hückel theory for the thermodynamic properties of bulk solutions containing point charges. Furthermore, it is shown that the pointcharge electrolyte model is not valid at low temperatures or high densities.

For a bulk system with no applied external potential, the ion density and the electric potential are uniform throughout the system [i.e., $\bar{\psi}(\mathbf{r})=0$, which is consistent with Eq. (19)]. Because the density does not depend on position, Eq. (21) can be rewritten as

$$
\mathcal{K}^{\text {bulk }}\left(\mathbf{r}, \mathbf{r}^{\prime}\right)=\delta\left(\mathbf{r}-\mathbf{r}^{\prime}\right) \frac{\epsilon \kappa_{\text {bulk }}^{2}}{4 \pi}
$$

where $\kappa_{\text {bulk }}$ is an, as yet, undetermined constant that can be identifed as the inverse screening length,

$$
\kappa_{\text {bulk }}^{2}=\frac{4 \pi \beta}{\epsilon} \sum_{\alpha} q_{\alpha}^{2} \rho_{\alpha}^{\text {bulk }}
$$

The Green's function associated with the screening function given in Eq. (22) is

$$
G_{\mathcal{K}}^{\text {bulk }}\left(\mathbf{r}, \mathbf{r}^{\prime}\right)=\frac{e^{-\kappa_{\text {bulk }}\left|\mathbf{r}-\mathbf{r}^{\prime}\right|}}{\epsilon\left|\mathbf{r}-\mathbf{r}^{\prime}\right|}
$$

and the corresponding expression for the grand partition function is

$$
\ln Z_{G}^{\text {bulk }}[\gamma] \geqslant V \sum_{\alpha} \lambda_{\alpha}^{-d} e^{\gamma_{\alpha}+\beta q_{\alpha}^{2} \kappa_{\text {bulk }} / 2 \epsilon}-V \frac{\kappa_{\text {bulk }}^{3}}{24 \pi},
$$

where $V$ is the volume of the system. The Debye-Hückel theory corresponds to a local maximum of Eq. (25) where the ion densities are given by

$$
\rho_{\alpha}^{\text {bulk }}=\frac{\partial \ln Z_{G}^{\text {bulk }}}{\partial \gamma_{\alpha}}=\lambda_{\alpha}^{-d} e^{\gamma_{\alpha}+\beta q_{\alpha}^{2} \kappa_{\text {bulk }} / 2 \epsilon},
$$

which leads to the standard expression for the chemical potential,

$$
\gamma_{\alpha}=\ln \rho_{\alpha}^{\text {bulk }} \lambda_{\alpha}^{d}-\frac{\beta q_{\alpha}^{2} \kappa_{\text {bulk }}}{2 \epsilon} .
$$

According to the variational principle, the value of $\kappa_{\text {bulk }}$ is determined from maximizing Eq. (25). Because this expression is greatest for an infinite value of $\kappa_{\text {bulk }}$, the DebyeHückel result is only a metastable state for the point-charge model. The stable solution corresponds to a state with infinite electrolyte density. This solution is an artifact of neglecting the finite size of the ions. However, as discussed below, the point-charge model still remains useful for sufficiently low density or high temperature where the details of the shortranged interactions between ions (e.g., hard-sphere diameter) do not strongly influence the maximum that corresponds to the Debye-Hückel result.

We can investigate the importance of a short-range repulsion in the theory by setting a cutoff wave vector $\Lambda$, which roughly corresponds to an inverse hard-core diameter (i.e., $\Lambda \sim 2 \pi / \sigma$, where $\sigma$ is roughly the range of the shortranged repulsive interactions). The Green's function in this case is

$$
G_{\mathcal{K}}\left(\mathbf{r}, \mathbf{r}^{\prime}\right)=\int_{0}^{\Lambda} \frac{4 \pi p^{2} d p}{(2 \pi)^{3}} e^{-i \mathbf{p} \cdot(\mathbf{r}-\mathbf{r})} \frac{4 \pi / \epsilon}{p^{2}+\kappa^{2}} .
$$

For the case of a symmetric electrolyte where the ions have a charge of either $+q$ or $-q$, the grand partition function is given by

$$
\begin{aligned}
\ln Z_{G}^{\text {bulk }}[\gamma] \geqslant & V \lambda_{+}^{-d} \exp \left(\gamma_{+}+\frac{\beta q^{2} \kappa}{2 \epsilon} \frac{\arctan (\Lambda / \kappa)}{\pi / 2}\right) \\
& +V \lambda_{-}^{-d} \exp \left(\gamma_{-}+\frac{\beta q^{2} \kappa}{2 \epsilon} \frac{\arctan (\Lambda / \kappa)}{\pi / 2}\right) \\
& -\frac{V \kappa^{3}}{24 \pi}\left[\arctan \frac{\Lambda}{\kappa}+\left(\frac{\Lambda}{\kappa}\right)^{3} \ln \left(1+\frac{\kappa^{2}}{\Lambda^{2}}\right)-\frac{\Lambda}{\kappa}\right] \frac{2}{\pi},
\end{aligned}
$$

where subscripts + and - refer to cations and anions, respectively. Once again, the value of $\kappa_{\text {bulk }}$ is determined by maxi- 
mizing the grand partition function; this leads to

$$
\left(\kappa l_{B}\right)^{2}=8 \pi \bar{\rho} l_{B}^{3} \exp \left(\frac{\kappa l_{B}}{2} \frac{\arctan (\Lambda / \kappa)}{\pi / 2}\right),
$$

where $l_{B}=\beta q^{2} / \epsilon$ is the Bjerrum length, which is the distance at which the Coulombic interaction between two ions becomes comparable to their thermal energy, and $\bar{\rho}=\left(\lambda_{+}^{-d} e^{\gamma_{+}}\right.$ $\left.+\lambda_{-}^{-d} e^{\gamma_{-}}\right) / 2$ is the mean fugacity coefficient. The standard expression for the inverse screening length is recovered (i.e., $\kappa^{2}=8 \pi \rho l_{B}$ ) by noting that

$$
\rho=\lambda_{ \pm}^{-d} e^{\gamma_{ \pm}} \exp \left(\frac{\kappa l_{B}}{2} \frac{\arctan (\Lambda / \kappa)}{\pi / 2}\right) .
$$

When $\bar{\rho} l_{B}^{3} \ll 1$, Eq. (29) has three roots. The middle root corresponds to a local minimum in the grand partition function and, thus, corresponds to an unstable state. The upper root corresponds to a metastable state and depends strongly on the value of the cutoff wave vector $\Lambda$. The lowest root corresponds to the equilibrium system and depends only weakly on the cutoff. Its value is given approximately by $\kappa_{\text {bulk }}^{2} \approx 8 \pi \bar{\rho} l_{B}$.

As the value of $\bar{\rho} l_{B}^{3}$ increases, the Coulombic coupling between ions becomes stronger. When $\bar{\rho} l_{B}^{3} \geqslant 2 e^{-2}$ [which corresponds to $\kappa l_{B} \geqslant 4$ or to a density of $\left.\rho l_{B}^{3} \geqslant 1 /(2 \pi)\right]$, the lower two roots of Eq. (29) vanish. The value of the remaining root depends strongly on the value of the cutoff wave vector $\Lambda$ indicating that the physics of the system is influenced by the short-ranged repulsive interactions which prevent the ions from overlapping. According to this analysis, at very low temperatures [where $\left.\rho l_{B}^{3} \geqslant 1 /(2 \pi)\right]$ the point-charge model is not valid even when the volume fraction occupied by the ions is vanishingly small.

\section{INTERFACIAL SYSTEMS}

In this section, we focus on electrolyte systems near a dielectric interface. The charged particles are immersed in a semi-infinite continuum solvent of dielectric constant $\epsilon$. On the other side of the interface, there is a continuum material of dielectric constant $\epsilon^{\prime}$ which does not contain electrolytes.

\section{A. Trial Green's function}

In order to evaluate the properties of the system, we need to solve the variational equation Eq. (21) to determine the function $\mathcal{K}$ and the associated Green's function $G_{\mathcal{K}}$. Unfortunately, determining the Green's function for an arbitrary choice of $\mathcal{K}$ is a difficult problem. Thus, to simplify the analysis, we restrict ourselves to forms of the function $\mathcal{K}$ such that the Green's function can be evaluated. The class of functions that we examine are

$$
\mathcal{K}\left(\mathbf{r}, \mathbf{r}^{\prime}\right)= \begin{cases}\frac{\epsilon \kappa^{2}}{4 \pi} \delta\left(\mathbf{r}-\mathbf{r}^{\prime}\right) & \text { inside } \epsilon \\ 0 & \text { inside } \epsilon^{\prime},\end{cases}
$$

where $\kappa$ is an, as yet, unknown constant, which corresponds to an inverse screening length. Physically, this choice of $\mathcal{K}$ corresponds to a system with no electrolyte present in the region $\epsilon^{\prime}$ and uniform screening in region $\epsilon$. The value of $\kappa$ is chosen so that the grand partition function is maximized. In this case, the variational condition Eq. (21) is replaced by the following condition:

$$
\frac{\partial \ln Z_{G}}{\partial \kappa^{2}}=0 \text {. }
$$

\section{B. Interfacial tension}

The interfacial free energy is related to the grand partition function of the interfacial system minus that of a bulk solution of the same geometry, temperature, and component chemical potentials. To simplify the analysis, symmetric electrolytes are considered with only electrostatic interactions (i.e., ideal-gas reference system) and without any fixed external charges. For this case, the electric potential in the interfacial system is uniform $[\bar{\psi}(\mathbf{r})=0]$ because there is no mechanism for the preferential interfacial adsorption of one electrolyte species over the other. The grand partition function of the interfacial system is then given by

$$
\begin{aligned}
\ln Z_{G}[\gamma, \Sigma] \geqslant & \sum_{\alpha} \lambda_{\alpha}^{-d} e^{\gamma_{\alpha}} \int d \mathbf{r} e^{-\left(\beta q_{\alpha}^{2} / 2\right) \Delta G_{\mathcal{K}}(\mathbf{r}, \mathbf{r})} \\
& -\frac{1}{2} \int_{0}^{1} d \zeta \operatorname{Tr} \mathcal{K}\left(G_{\zeta \mathcal{K}}-G_{\mathcal{K}}\right) \\
= & V\left[-\frac{\kappa^{3}}{24 \pi}+\sum_{\alpha} \rho_{\alpha}^{\text {bulk }}\right] \\
& -\frac{1}{2} \int_{0}^{1} d \zeta \operatorname{Tr} \mathcal{K}\left(\delta G_{\zeta \mathcal{K}}-\delta G_{\mathcal{K}}\right)+\sum_{\alpha} \rho_{\alpha}^{\text {bulk }} \\
& \times \int d \mathbf{r}\left\{\exp \left[-\frac{\beta q_{\alpha}^{2}}{2} \delta G_{\mathcal{K}}(\mathbf{r}, \mathbf{r})\right]-1\right\},
\end{aligned}
$$

where $\delta G_{\mathcal{K}}=G_{\mathcal{K}}-G_{\mathcal{K}}^{\text {bulk. }}$. The first term on the left side of Eq. (33) corresponds to the grand partition function of the bulk system and is on the order of the volume $V$, while the other terms are only on the order of the interfacial area $A$. Consequently, to order $1 / L=A / V$, the variational equations are identical to those of the bulk system. Because the electrolyte systems are chosen to be infinite, the value of the inverse screening length in the interfacial system is equal to that for the bulk system (i.e., $\kappa=\kappa_{\text {bulk }}$ ), as given in Eq. (29).

The excess interfacial tension $\sigma$ is given by the difference in the grand partition function of the interfacial system and that of the bulk system:

$$
\begin{aligned}
\beta \sigma A= & -\left\{\ln Z_{G}[\gamma, \Sigma]-\ln Z_{G}^{\text {bulk }}[\gamma]\right\} \\
= & \frac{1}{2} \int_{0}^{1} d \zeta \operatorname{Tr} \mathcal{K}\left(\delta G_{\zeta \mathcal{K}}-\delta G_{\mathcal{K}}\right) \\
& -2 \rho^{\text {bulk }} \int d \mathbf{r}\left[e^{-\left(\beta q^{2} / 2\right) \delta G(\mathbf{r}, \mathbf{r})}-1\right],
\end{aligned}
$$

and the electrolyte density profile about the dielectric interface to one loop order is given by following the functional derivative: ${ }^{32}$ 


$$
\rho_{\alpha}(\mathbf{r})=\frac{\delta \ln Z_{G}}{\delta \gamma_{\alpha}(\mathbf{r})}=\rho_{\alpha}^{\text {bulk }} \exp \left[-\frac{\beta q_{\alpha}^{2}}{2} \delta G_{\mathcal{K}}(\mathbf{r}, \mathbf{r})\right] .
$$

Accordingly, the term $\left(q_{\alpha}^{2} / 2\right) \delta G_{\mathcal{K}}(\mathbf{r}, \mathbf{r})$ can be identified as the potential of the mean force between the ion and the interface, which is the reversible work to move an ion from infinitely far from the interface to a position $\mathbf{r} .{ }^{19}$

\section{PLANAR DIELECTRIC INTERFACE}

The Green's function for a planar interface is given by ${ }^{19}$

$$
\begin{aligned}
G_{\mathcal{K}}\left(\mathbf{r}, \mathbf{r}^{\prime}\right)= & \frac{2 \pi}{\epsilon} \int_{\mathbf{p}} \frac{1}{\left(p^{2}+\kappa^{2}\right)^{1 / 2}}\left[e^{-\left(p^{2}+\kappa^{2}\right)^{1 / 2}\left|z-z^{\prime}\right|}\right. \\
& \left.-\Delta(p ; \eta, \kappa) e^{-\left(p^{2}+\kappa^{2}\right)^{1 / 2}\left(z+z^{\prime}\right)}\right] e^{-i p_{x}\left(x-x^{\prime}\right)-i p_{y}\left(y-y^{\prime}\right)} \\
= & \frac{e^{-\kappa\left|\mathbf{r}-\mathbf{r}^{\prime}\right|}}{\epsilon\left|\mathbf{r}-\mathbf{r}^{\prime}\right|}+\delta G_{\mathcal{K}}\left(\mathbf{r}, \mathbf{r}^{\prime}\right),
\end{aligned}
$$

where $z$ is the normal distance from the dielectric interface into the electrolyte-containing medium, $\eta=\epsilon^{\prime} / \epsilon$ is the dielectric constant ratio,

$$
\begin{aligned}
\delta G_{\mathcal{K}}\left(\mathbf{r}, \mathbf{r}^{\prime}\right)= & -\frac{2 \pi}{\epsilon} \int_{\mathbf{p}} \frac{\Delta(p ; \eta, \kappa)}{\left(p^{2}+\kappa^{2}\right)^{1 / 2}} \\
& \times e^{-\left(p^{2}+\kappa^{2}\right)^{1 / 2}\left(z+z^{\prime}\right)} e^{-i p_{x}\left(x-x^{\prime}\right)-i p_{y}\left(y-y^{\prime}\right)},
\end{aligned}
$$

and

$$
\Delta(p ; \eta, \kappa)=\frac{\eta p-\sqrt{p^{2}+\kappa^{2}}}{\eta p+\sqrt{p^{2}+\kappa^{2}}} .
$$

The first term in Eq. (36) is the Green's function for a bulk electrolyte, while the second term accounts for the influence of the dielectric interface. The resulting ion-interface potential of the mean force is given by

$$
\begin{aligned}
\delta G_{\mathcal{K}}(\mathbf{r}, \mathbf{r})= & -\frac{1}{\epsilon} \int_{0}^{\infty} d p \frac{p \Delta(p)}{\left(p^{2}+\kappa^{2}\right)^{1 / 2}} e^{-2\left(p^{2}+\kappa^{2}\right)^{1 / 2} z} \\
= & -\left(\frac{\eta-1}{\eta+1}\right) \frac{e^{-2 \kappa z}}{2 \epsilon z} \\
& -\frac{2 \eta}{\eta+1} \frac{\kappa}{\epsilon} \int_{1}^{\infty} d x \frac{\sqrt{x^{2}-1}-x}{\eta \sqrt{x^{2}-1}+x} e^{-2 x \kappa z} .
\end{aligned}
$$

The initial term of Eq. (39) is the screened image charge interaction between the dielectric wall and an ion that was first used by Onsager and Samaras. This interaction can be attractive if $\eta>1$ as occurs with an electrolyte solution next to a metal surface or repulsive if $\eta<1$ when the surface next to the electrolyte solution has a lower dielectric than water. The second term in Eq. (39) arises because the electrolyte has been confined to the space where $z>0$. This term is always repulsive because ions prefer to be far from the interface where they are entirely surrounded by other ions. Because of this confinement effect, the ion-interface potential of the mean force is still respulsive even in the absence of a dielectric interface (i.e., $\eta=1$ ). This general behavior for $\delta G_{\mathcal{K}}$ has also been observed by Dean and Horgan, ${ }^{21}$ who used a one-loop approximation for evaluating Eq. (5).

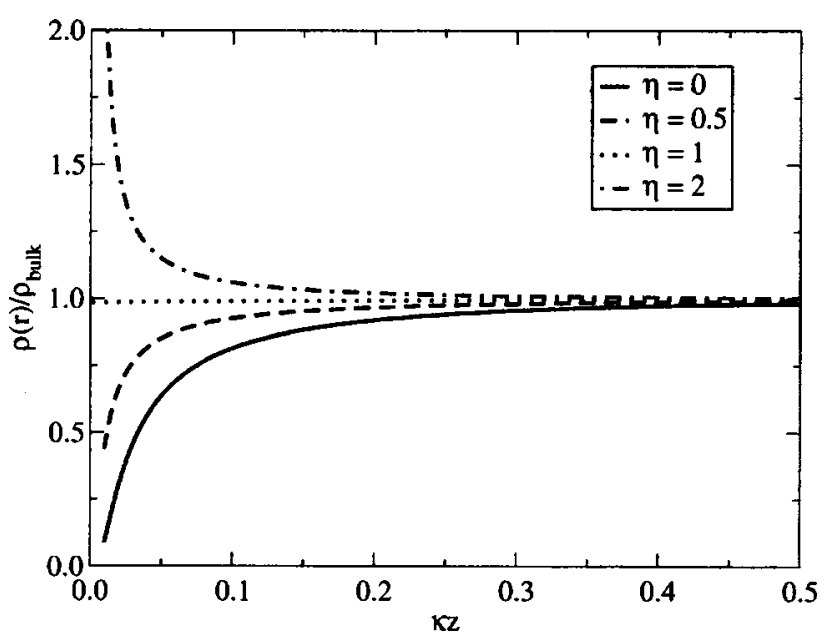

FIG. 1. Ion density distribution for a symmetric electrolyte near a flat interface with $\kappa l_{B}=1$ and (i) $\eta=0$ (solid line), (ii) $\eta=0.5$ (dashed line), (iii) $\eta=1$ (dotted line), and (iv) $\eta=2$ (dashed-dotted line).

In Fig. 1, the ion density is plotted as a function of the distance from the interface at various values of $\eta$. As mentioned above, when $\eta<1$, the ion-interface potential of the mean force is repulsive and the ions are preferentially desorbed from the interface. When $\eta \gg 1$, the image charge interactions are attractive, and the ions adsorb preferentially to the interface. For the case where $\eta=1$, there are no image charge interactions. However, despite the absence of these repulsive interactions, slight preferential desorption of the ions from the wall is observed due to the confinement effect described above.

Evaluating the excess surface tension requires

$$
\begin{aligned}
& -\frac{1}{2} \int_{0}^{1} d \zeta \operatorname{Tr} \mathcal{K}\left(\delta G_{\zeta \mathcal{K}}-\delta G_{\mathcal{K}}\right) \\
& \quad=\frac{\kappa^{2} A}{16 \pi} \int_{0}^{1} d \zeta \int_{0}^{\infty} d p p\left[\frac{\Delta(p ; \eta, \sqrt{\zeta} \kappa)}{\left(p^{2}+\zeta \kappa^{2}\right)}-\frac{\Delta(p ; \eta, \kappa)}{\left(p^{2}+\kappa^{2}\right)}\right] \\
& \quad=\frac{\kappa^{2} A}{32 \pi}\left(\frac{\eta-1}{\eta+1}\right) .
\end{aligned}
$$

In Fig. 2, the excess surface tension $\sigma$ is plotted versus $\left(\kappa l_{B}\right)^{2}$ (dimensionless electrolyte concentration) for different values of $\eta$. As expected, the excess surface tension increases with increasing ion concentration. This effect is diminished as the dielectric constant of the wall increases leading to a decrease in the screened image charge repulsion. Even when the dielectric constant of the wall and that of the solvent are the same (i.e., $\eta=1$ ), the excess surface tension is positive due to the slight exclusion of ions from the interface.

The inset in Fig. 2 shows the excess surface tension in experimentally accessible units, evaluated for a monovalent electrolyte in water at $25^{\circ} \mathrm{C}$ (where $l_{B} \approx 7 \AA$ ). This should be compared with an experimentally measured surface tension of a solution of sodium chloride which is given by $\sigma=1.6 c$, where $c$ is salt concentration in $\mathrm{mol} / \mathrm{L}$, and $\sigma$ is in $\mathrm{mN} / \mathrm{m}$. The results presented here underpredict the experimentally observed values, as has been previously observed with similar theories. ${ }^{33}$ The inability of the theory is attributed to using 


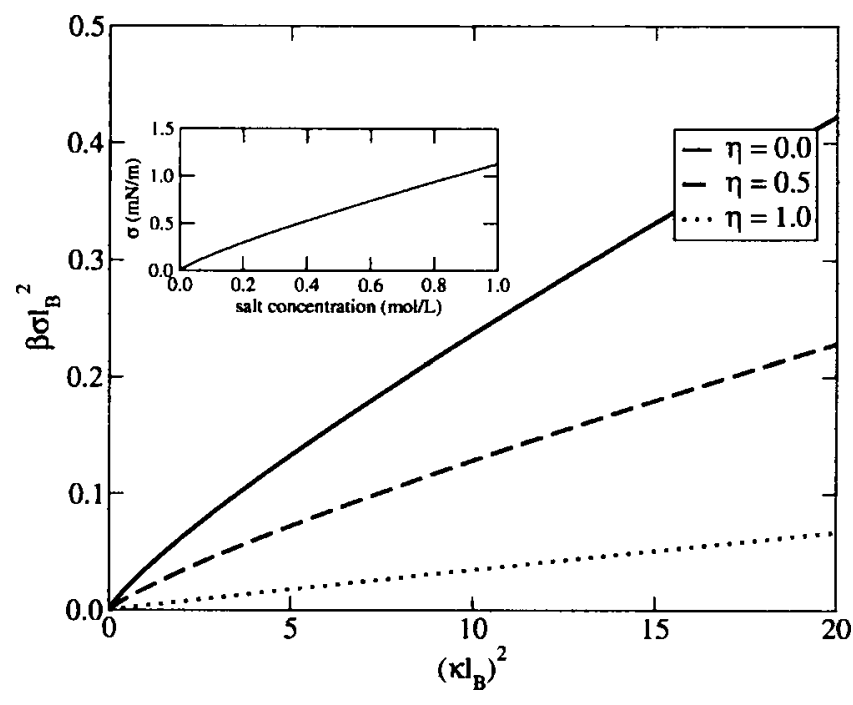

FIG. 2. Excess surface tension for a symmetric electrolyte at a planar dielectric interface with $\eta=0$ (solid line), $\eta=0.5$ (dashed line), and $\eta=1$ (dotted line). The inset is for the case where $\eta=0, l_{B}=7 \AA$, and $T=25^{\circ} \mathrm{C}$.

the bulk screening length near to the interface and to neglecting other interactions specific to the nature of the ion, such as ion excluded volume forces, ${ }^{11}$ ionic dispersion interactions, ${ }^{23}$ or to the structure-making or breaking ability of the ions. ${ }^{34}$

In the limit that $\eta \ll 1$, the expression for the excess interfacial tension $\sigma$ simplifies to

$$
\begin{aligned}
\beta \sigma & =\frac{\kappa^{2}}{32 \pi}-2 \rho \int_{0}^{\infty} d z\left[\exp \left(-\frac{\beta q^{2}}{4 \epsilon z} e^{-2 \kappa z}\right)-1\right] \\
& =\frac{\rho l_{B}}{2}\left[\frac{1}{2}-\frac{2}{\kappa l_{B}} \int_{0}^{\infty} d t \exp \left(-\frac{\kappa l_{B} e^{-t}}{2 t}\right)-1\right] .
\end{aligned}
$$

Expanding this expression at low electrolyte concentrations yields ${ }^{3}$

$$
\begin{aligned}
\beta \sigma \approx & \frac{\rho l_{B}}{2}\left[\left(\frac{3}{2}-2 \gamma_{E}-\ln \frac{\kappa l_{B}}{2}\right)\right. \\
& \left.-\left(\frac{\kappa l_{B}}{2}\right)\left(\ln \frac{\kappa l_{B}}{2}+\ln 2-2+2 \gamma_{E}\right)+\cdots\right],
\end{aligned}
$$

where $\gamma_{E} \approx 0.5772157$ is Euler's constant. The lowest order term is precisely the same as that obtained by Onsager and Samaras, ${ }^{3}$ as well as by Levin, who used an alternate method. ${ }^{33}$

\section{DIELECTRIC SPHERE IN AN ELECTROLYTE}

In this section, we consider a spherical particle of radius $R$ and dielectric constant $\epsilon^{\prime}$ immersed in an electrolytecontaining medium (with dielectric constant $\epsilon$ ). The electrolyte is restricted to the outside of the sphere. The Green's function for this problem is given by (see Appendix for details)

$$
G_{\mathcal{K}}\left(\mathbf{r}, \mathbf{r}^{\prime}\right)=\frac{e^{-\kappa\left|\mathbf{r}-\mathbf{r}^{\prime}\right|}}{\epsilon\left|\mathbf{r}-\mathbf{r}^{\prime}\right|}+\delta G_{\mathcal{K}}\left(\mathbf{r}, \mathbf{r}^{\prime}\right),
$$

where

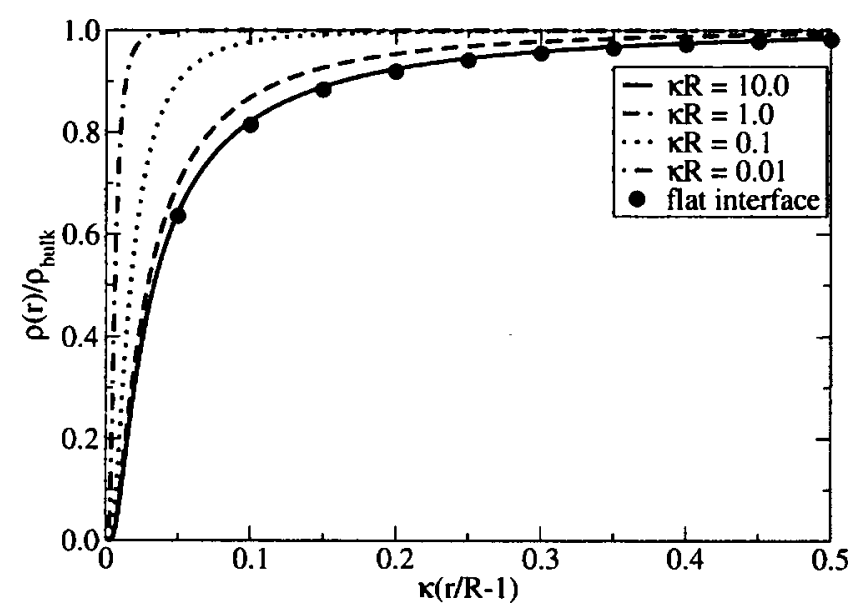

FIG. 3. Density profile for symmetric electrolytes near a dielectric sphere with $\eta=0, \kappa l_{B}=1$, and $\kappa R=0.01$ (dashed-dotted line), $\kappa R=0.1$ (dotted line), $\kappa R=1$ (dashed line), $\kappa R=10$ (solid line), and planar interface (circles).

$$
\begin{aligned}
\delta G_{\mathcal{K}}\left(\mathbf{r}, \mathbf{r}^{\prime}\right)= & -\frac{4 \pi \kappa}{\epsilon} \sum_{l m} k_{l}\left(\kappa r_{<}\right) k_{l}\left(\kappa r_{>}\right) \\
& \times D_{l}^{\text {out }}(\kappa R, 0, \eta) Y_{l m}(\theta, \phi) Y_{l m}^{*}\left(\theta^{\prime}, \phi^{\prime}\right),
\end{aligned}
$$

$\theta$ is the polar angle of position $\mathbf{r}, \phi$ is the azimuth angle of position $\mathbf{r}, \theta^{\prime}$ is the polar angle of position $\mathbf{r}^{\prime}, \phi^{\prime}$ is the azimuth angle of position $\mathbf{r}^{\prime}, r_{<}$is the radial distance of the position closer to the center of the sphere, and $r_{<}$is the radial distance of the position further from the center of the sphere. The function $Y_{l m}$ is a spherical harmonic function and $D_{l}^{\text {out }}$ is given by

$$
D_{l}^{\text {out }}(x, 0, \eta)=\frac{i_{l}(x)}{k_{l}(x)}\left[\frac{l \eta-x i_{l}^{\prime}(x) / i_{l}(x)}{l \eta-x k_{l}^{\prime}(x) / k_{l}(x)}\right] .
$$

The functions $i_{l}$ and $k_{l}$ are the modified spherical Bessel functions of the first kind and of the second kind, respectively.

The ion-interface potential of the mean force is given by

$$
\delta G_{\mathcal{K}}(\mathbf{r}, \mathbf{r})=-\frac{2 \kappa}{\epsilon} \sum_{l=0}^{\infty}(l+1 / 2) \kappa_{l}^{2}(\kappa r) D_{l}^{\text {out }}(\kappa R, 0, \eta)
$$

In the absence of screening by other ions (i.e., $\kappa=0$ ), the potential of the mean force reduces to the potential of a point charge with its image charges, ${ }^{13,35}$

$$
\delta G_{\mathcal{K}}(\mathbf{r}, \mathbf{r})=-\frac{2}{\epsilon r} \sum_{l=1}^{\infty} \frac{\eta-1}{\eta+1+1 / l}\left(\frac{R}{r}\right)^{2 l+1} .
$$

The density profile is determined from the ion-interface potential of the mean force [see Eq. (35)] which depends only on the ratio of the particle radius to the screening length $\kappa R$. The density profiles for different values of $\kappa R$ are plotted in Fig. 3 for the case where $\eta=0$. In the limit that $\kappa R \gg 1$, the correlation lengths in the solvent (as represented by the screening length) become small compared with the radius of curvature, and the expression for the self-energy reduces to that found for a planar dielectric interface [see Eq. (39)], with $z=r-R$ being the distance from the surface of the sphere. The effect of curvature is important when the radius 


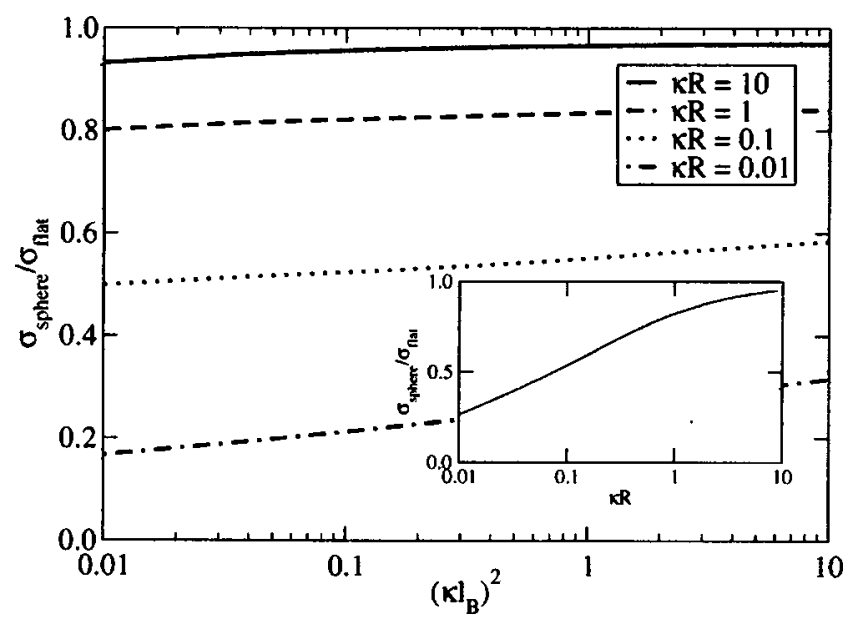

FIG. 4. Ratio of the excess surface tension for a dielectric sphere with $\epsilon^{\prime} \gg \epsilon$ immersed in a symmetric electrolyte and surface tension for a planar interface: $\kappa R=0.01$ (solid line), $\kappa R=0.1$ (dashed line), $\kappa R=1$ (dotted line), and $\kappa R=10$ (dashed-dotted line). The inset shows the variation of the excess surface tension with $\kappa R$ for $\kappa l_{B}=1$.

of the particle becomes small compared with the screening length $\kappa R<1$. For decreasing values of $\kappa R$, the magnitude of the screened image interaction is lowered resulting in a decreased amount of ion desorption. As in the case of the planar geometry, even when the two dielectric constants are identical, there is still an effective repulsion of the ions from the interface.

To evaluate the excess surface tension requires the following integral:

$$
\begin{aligned}
& -\frac{1}{2} \int_{0}^{1} d \zeta \operatorname{Tr} \mathcal{K}\left(\delta G_{\zeta \mathcal{K}}-\delta G_{\mathcal{K}}\right) \\
& =-\sum_{l=0}^{\infty}(l+1 / 2) \int_{0}^{\kappa R} d \zeta \zeta\left\{\zeta D _ { l } ^ { \text { out } } ( \zeta , 0 , \eta ) \left[k_{l}^{2}(\zeta)\right.\right. \\
& \left.\quad-k_{l-1}(\zeta) k_{l+1}(\zeta)\right]-(\kappa R) D_{l}^{\text {out }}(\kappa R, 0, \eta)\left[k_{l}^{2}(\kappa R)\right. \\
& \left.\left.\quad-k_{l-1}(\kappa R) k_{l+1}(\kappa R)\right]\right\} .
\end{aligned}
$$

This expression was obtained by noting that $\mathcal{K}$ vanishes inside the dielectric sphere and is a constant outside. In addition, we use the second Lommel integral: ${ }^{36}$

$$
\int_{a}^{\infty} d x x^{2} k_{l}^{2}(x)=-\frac{a}{2}\left[k_{l}^{2}(a)-k_{l-1}(a) k_{l+1}(a)\right] .
$$

In Fig. 4 , the cavity correction factor $\sigma_{\text {sphere }} / \sigma_{\text {flat }}$ (which is a measure of the influence of curvature on interfacial tension) is plotted as a function of $\left(\kappa l_{B}\right)^{2}$ (ion concentration) at various values of $\kappa R$ for the case where $\eta=0$. The cavity correction factor is only a weak function of ion concentration when evaluated at constant $\kappa R$. The correction factor is a much stronger function of $\kappa R$, as also demonstrated by the inset of Fig. 4 where it is plotted versus $\kappa R$ for $\kappa l_{B}=1$. In the limit that the particle radius becomes large with respect to the screening length (i.e., $\kappa R \gg 1$ ), $\sigma_{\text {sphere }} / \sigma_{\text {flat }} \rightarrow 1$ as expected because the curved surface appears flat on the scale of the correlation lengths in the solvent. As $\kappa R$ is decreased, the cavity correction factor is lowered. This effect can be attrib- uted to a smaller amount of ion desorption from the curved surface than from the flat surface, which is in agreement with the findings of Groenewold ${ }^{14}$ for weakly curved dielectric interfaces.

It is insightful to determine the cavity correction factor for a typical protein, such as lysozyme (with a radius of $15 \AA$ ) dissolved in an aqueous solution of monovalent ions. For salt concentrations ranging from 0.1 , to $1.0 \mathrm{M}$ the values of $\kappa R$ range from about 1.5 to 5 , and according to Fig. 4 , this would correspond to correction factors between 0.8 and 0.95 , respectively.

The microscopic equivalent of electrolyte adsorption at an interface is the protein-salt preferential interaction parameter-the difference in the salt molality in the domain of the protein over that in bulk. Arakawa and Timasheff ${ }^{1}$ have measured this parameter for lysozyme or bovine serum albumin (BSA) in various salt solutions. The experimentally observed parameters are within a factor of about 0.7 of those values calculated assuming the protein is a low dielectric body with no curvature. In this case, the preferential interaction parameter can be calculated from the molal surfacetension increment of the salt. The reason that there is less exclusion of salt about a protein than as predicted using this assumption was attributed to specific interactions between the salt and the protein surface.

We have shown that increasing the curvature of the protein surface will slightly decrease salt exclusion. While this effect is not large enough to entirely account for the discrepancy with the experimentally observed preferential interaction parameters, it does indicate that the dominant forces that control the exclusion of the salt about the protein surface are similar to those that control the distribution of ions about a low dielectric cavity. In dilute electrolyte solutions, the exclusion of the salt is driven primarily by (geometrydependent) image charge interactions, which the above theory incorporates. For more concentrated salt solutions, however, other factors need to be included such as ion excluded volume, ${ }^{11}$ ion dispersion forces, ${ }^{23}$ or forces resulting from the structure-making or breaking ability of the ion. ${ }^{34}$

\section{CONCLUSIONS}

A variational theory was developed and applied to study the properties of electrolyte solutions at dielectric interfaces. The particular cases of planar and spherical geometries were examined. The advantage of this approach over the standard Poisson-Boltzmann equation is that it includes ion-ion correlation effects. Also, because we work directly with a free energy, the need to integrate the Gibbs adsorption equation is avoided and thermodynamic consistency is ensured.

For the planar case, the ions preferentially desorb from the interface. There are two contributions to the potential of the mean force between an ion and the interface: a screened image charge interaction and a repulsive force originating from exclusion of the electrolytes from the dielectric wall. The lower the dielectric constant of the interface, the more strongly the ions desorb, and consequently, the larger the excess surface tension. Even when the dielectric constant of the interface is the same as in the bulk, the ions still desorb 
from the interface, resulting in a positive excess surface tension. At low electrolyte concentrations, the excess surface tension predicted by the variational theory reduces to the Onsager-Samaras limiting law. ${ }^{3}$

For spherical dielectric particles, the excess surface tension decreases as the particle radius decreases. In the limit that the radius of the sphere becomes much larger than the Debye screening length, the properties of the system reduces to that of a planar dielectric interface.

In this work, we only have examined symmetric electrolytes. If there are any differences between the negative and positive charge carriers (e.g., a different valence or specific interaction with the interface), then this asymmetry will lead to charge separation and the generation of a potential difference between the dielectric particle and the bulk electrolyte solution. In addition, we have neglected the presence of nonelectrostatic interaction between particles. These interactions can be included by using a reference state grand partition function $Z_{G}^{\text {ref }}$ other than the ideal-gas reference state (e.g., the Carnahan-Starling equation of $\operatorname{state}^{37}$ can be used to include excluded volume interactions ${ }^{24}$ ). This approach can also be used to include specific interactions between the ions and the interface. This is especially important to study the Hofmeister effect where the surface tension depends on the specific nature of the ion. These nonelectrostatic interactions can also lead to the enhanced adsorption of highly polarizable ions at low dielectric interfaces. ${ }^{38}$ Consequently, other ion specific interations such as ion-dispersion interactions and effects due to ion hydration need to be included in surface tension models to better understand the Hofmeister effect.

\section{APPENDIX: GREEN'S FUNCTION FOR THE STURM-LIOUVILLE PROBLEM}

In this Appendix, we develop the Green's function associated with the screening function given in Eq. (31) for spherical geometries. The Green's function $G_{\mathcal{K}}$ is given by the solution of

$$
\frac{\epsilon(r)}{4 \pi}\left[-\frac{1}{\epsilon(r)} \nabla \cdot \epsilon(r) \nabla+\kappa^{2}(r)\right] G_{\mathcal{K}}\left(\mathbf{r}, \mathbf{r}^{\prime}\right)=\delta^{d}\left(\mathbf{r}-\mathbf{r}^{\prime}\right),
$$

where $\epsilon(r)$ is the spatially varying dielectric constant, and $\kappa(r)$ is the spatially varying inverse screening length. The Green's function can be expanded in a series involving the spherical harmonics $Y_{l m}$,

$$
G_{\mathcal{K}}\left(\mathbf{r}, \mathbf{r}^{\prime}\right)=\sum_{l m} g_{l}\left(r, r^{\prime}\right) Y_{l m}(\theta, \phi) Y_{l m}^{*}\left(\theta^{\prime}, \phi^{\prime}\right) .
$$

Substituting this expansion into the Green's function equation yields

$$
\begin{aligned}
& \frac{\epsilon(r)}{4 \pi r^{2}}\left[-\frac{1}{\epsilon(r)} \frac{\partial}{\partial r} r^{2} \epsilon(r) \frac{\partial}{\partial r}+l(l+1)+r^{2} \kappa^{2}(r)\right] g_{l}\left(r, r^{\prime}\right) \\
& =\frac{1}{r^{2}} \delta\left(r-r^{\prime}\right) .
\end{aligned}
$$

For the system considered in this work, the dielectric con- stant and the screening length are constant within the droplet and outside the droplet, although they are discontinuous across the two regions. If we focus on one of these regions, the Green's function problem reduces to

$$
\frac{\epsilon}{4 \pi r^{2}}\left[-\frac{\partial}{\partial r} r^{2} \frac{\partial}{\partial r}+l(l+1)+r^{2} \kappa^{2}\right] g_{l}\left(r, r^{\prime}\right)=\delta\left(r-r^{\prime}\right) \text {. }
$$

For this one-dimensional problem, the Green's function is simply given by ${ }^{39}$

$$
g_{l}\left(r, r^{\prime}\right)=-\frac{1}{C}\left\{\begin{array}{l}
u(r) v\left(r^{\prime}\right) \text { for } r<r^{\prime} \\
u\left(r^{\prime}\right) v(r) \text { for } r>r^{\prime},
\end{array}\right.
$$

where $u$ and $v$ are solutions of the corresponding homogeneous differential equation, which is

$$
\frac{\epsilon}{4 \pi r^{2}}\left[-\frac{d}{d r} r^{2} \frac{d}{d r}+l(l+1)+r^{2} \kappa^{2}\right] y(r)=0,
$$

and its solutions are linear combinations of modified, spherical Bessel functions $i_{l}(r)$ and $k_{l}(r)$, which are defined as

$$
\begin{aligned}
& i_{l}(x)=\left(\frac{\pi}{2 x}\right)^{1 / 2} I_{l+1 / 2}(x)=\frac{(x / 2)^{l}}{\Gamma(l+1)} \frac{1}{2} \int_{-1}^{1} d t\left(1-t^{2}\right)^{l} e^{-x t}, \\
& k_{l}(x)=\left(\frac{2}{\pi x}\right)^{1 / 2} K_{l+1 / 2}(x)=\frac{(x / 2)^{l}}{\Gamma(l+1)} \int_{1}^{\infty} d t\left(t^{2}-1\right)^{l} e^{-x t},
\end{aligned}
$$

where $I_{l}$ and $K_{l}$ are the modified Bessel functions of the first and second kinds, respectively.

The general solution of the homogeneous differential equation can be written as a linear combination of the two independent solutions,

$$
\begin{aligned}
& u(r)=A_{u} i_{l}(\kappa r)+B_{u} k_{l}(\kappa r), \\
& v(r)=A_{v} i_{l}(\kappa r)+B_{v} k_{l}(\kappa r),
\end{aligned}
$$

where $A_{u}, B_{u}, A_{v}$, and $B_{v}$ are constants chosen such that the solution $u$ satisfies the lower boundary condition, and the solution $v$ satisfies the upper boundary condition.

Inside the sphere (i.e., $r<R$ ), it is assumed that the inverse Debye screening length is $\kappa_{2}$ and the dielectric constant is $\epsilon_{2}$. Outside the sphere, it is assumed that the inverse Debye screening length is $\kappa_{1}$ and the dielectric constant is $\epsilon_{1}$.

$$
\begin{aligned}
& u(r)= \begin{cases}A_{u}^{(2)} i_{l}\left(\kappa_{2} r\right) & r<R \\
A_{u}^{(1)} i_{l}\left(\kappa_{1} r\right)+B_{u}^{(1)} k_{l}\left(\kappa_{1} r\right) & r>R,\end{cases} \\
& v(r)= \begin{cases}A_{v}^{(2)} i_{l}\left(\kappa_{2} r\right)+B_{v}^{(2)} k_{l}\left(\kappa_{2} r\right) & r<R \\
B_{v}^{(1)} k_{l}\left(\kappa_{1} r\right) & r>R .\end{cases}
\end{aligned}
$$

The functions $u$ and $v$ must be continuous. In addition, the displacement vector must also be continuous. Applying these conditions to the function $u$ yields the following equations:

$$
A_{u}^{(1)} i_{l}\left(\kappa_{1} R\right)+B_{u}^{(1)} k_{l}\left(\kappa_{1} R\right)=A_{u}^{(2)} i_{l}\left(\kappa_{2} R\right),
$$




$$
A_{u}^{(1)} \epsilon_{1} \kappa_{1} i_{l}^{\prime}\left(\kappa_{1} R\right)+B_{u}^{(1)} \epsilon_{1} \kappa_{1} k_{l}^{\prime}\left(\kappa_{1} R\right)=A_{u}^{(2)} \epsilon_{2} \kappa_{2} i_{l}^{\prime}\left(\kappa_{2} R\right)
$$

For this scale, we choose $A_{u}^{(2)}=1$. Using the following identity: ${ }^{39}$

$$
i_{l}(x) k_{l}^{\prime}(x)-i_{l}^{\prime}(x) k_{l}(x) \equiv-\frac{1}{x^{2}},
$$

these equations can be inverted to give explicit expressions for $A_{u}^{(1)}$ and $B_{u}^{(1)}$,

$$
\begin{aligned}
A_{u}^{(1)}= & -\frac{\left(\kappa_{1} R\right)^{2}}{\epsilon_{1} \kappa_{1}}\left[\epsilon_{1} \kappa_{1} k_{l}^{\prime}\left(\kappa_{1} R\right) i_{l}\left(\kappa_{2} R\right)\right. \\
& \left.-\epsilon_{2} \kappa_{2} i_{l}^{\prime}\left(\kappa_{2} R\right) k_{l}\left(\kappa_{1} R\right)\right], \\
B_{u}^{(1)}= & \frac{\left(\kappa_{1} R\right)^{2}}{\epsilon_{1} \kappa_{1}}\left[\epsilon_{1} \kappa_{1} i_{l}^{\prime}\left(\kappa_{1} R\right) i_{l}\left(\kappa_{2} R\right)-\epsilon_{2} \kappa_{2} i_{l}^{\prime}\left(\kappa_{2} R\right) i_{l}\left(\kappa_{1} R\right)\right] .
\end{aligned}
$$

Similarly for the function $v$, we have

$$
\begin{aligned}
& B_{v}^{(1)} k_{l}\left(\kappa_{1} R\right)=A_{v}^{(2)} i_{l}\left(\kappa_{2} R\right)+B_{v}^{(2)} k_{l}\left(\kappa_{2} R\right), \\
& B_{v}^{(1)} \epsilon_{1} \kappa_{1} k_{l}^{\prime}\left(\kappa_{1} R\right)=A_{v}^{(2)} \epsilon_{2} \kappa_{2} i_{l}^{\prime}\left(\kappa_{2} R\right)+B_{v}^{(2)} \epsilon_{2} \kappa_{2} k_{l}^{\prime}\left(\kappa_{2} R\right) .
\end{aligned}
$$

Choosing the scale of $v$ by setting $B_{v}^{(1)}=1$, the solutions of the above equations are

$$
\begin{aligned}
A_{v}^{(2)}= & -\frac{\left(\kappa_{2} R\right)^{2}}{\epsilon_{2} \kappa_{2}}\left[\epsilon_{2} \kappa_{2} k_{l}^{\prime}\left(\kappa_{2} R\right) k_{l}\left(\kappa_{1} R\right)\right. \\
& \left.-\epsilon_{1} \kappa_{1} k_{l}\left(\kappa_{2} R\right) k_{l}^{\prime}\left(\kappa_{1} R\right)\right], \\
B_{v}^{(2)}= & \frac{\left(\kappa_{2} R\right)^{2}}{\epsilon_{2} \kappa_{2}}\left[\epsilon_{2} \kappa_{2} i_{l}^{\prime}\left(\kappa_{2} R\right) k_{l}\left(\kappa_{1} R\right)-\epsilon_{1} \kappa_{1} i_{l}\left(\kappa_{2} R\right) k_{l}^{\prime}\left(\kappa_{1} R\right)\right] .
\end{aligned}
$$

The constant $C$ is related ${ }^{39}$ to the Wronskian of Eq. (A6),

$$
\begin{aligned}
C & =p(x) W(x) \\
& =p(x)\left[u(x) v^{\prime}(x)-u^{\prime}(x) v(x)\right] \\
& =\frac{R^{2}}{4 \pi}\left[\epsilon_{1} \kappa_{1} k_{l}^{\prime}\left(\kappa_{1} R\right) i_{l}\left(\kappa_{2} R\right)-\epsilon_{2} \kappa_{2} i_{l}^{\prime}\left(\kappa_{2} R\right) k_{l}\left(\kappa_{1} R\right)\right] .
\end{aligned}
$$

The Green's function is then given by

$$
\begin{aligned}
g_{l}\left(r, r^{\prime}\right)= & -\frac{4 \pi}{R^{2}} \\
& \times \frac{\left[A_{u} i_{l}\left(\kappa r_{<}\right)+B_{u} k_{l}\left(\kappa r_{<}\right)\right]\left[A_{v} i_{l}\left(\kappa r_{>}\right)+B_{v} k_{l}\left(\kappa r_{>}\right)\right]}{\epsilon_{1} \kappa_{1} k_{l}^{\prime}\left(\kappa_{1} R\right) i_{l}\left(\kappa_{2} R\right)-\epsilon_{2} \kappa_{2} i_{l}^{\prime}\left(\kappa_{2} R\right) k_{l}\left(\kappa_{1} R\right)},
\end{aligned}
$$

where $r_{>}\left(r_{<}\right)$is the larger (lesser) of the two radial distances $r$ and $r^{\prime}$. When both the vectors $\mathbf{r}$ and $\mathbf{r}^{\prime}$ lie outside the sphere, the Green's function reduces to

$$
\begin{aligned}
g_{l}\left(r, r^{\prime}\right)= & \frac{4 \pi \kappa_{1}}{\epsilon_{1}}\left[i_{l}\left(\kappa_{1} r_{<}\right)\right. \\
& \left.-D_{l}^{\text {out }}\left(\kappa_{1} R, \kappa_{2} R, \eta\right) k_{l}\left(\kappa_{1} r_{<}\right)\right] k_{l}\left(\kappa_{1} r_{>}\right),
\end{aligned}
$$

where

$$
\begin{aligned}
D_{l}^{\text {out }}\left(x_{1}, x_{2}, \eta\right)= & \frac{i_{l}\left(x_{1}\right)}{k_{l}\left(x_{1}\right)} \\
& \times\left[\frac{\epsilon_{2} x_{2} i_{l}^{\prime}\left(x_{2}\right) / i_{l}\left(x_{2}\right)-\epsilon_{1} x_{1} i_{l}^{\prime}\left(x_{1}\right) / i_{l}\left(x_{1}\right)}{\epsilon_{2} x_{2} i_{l}^{\prime}\left(x_{2}\right) / i_{l}\left(x_{2}\right)-\epsilon_{1} x_{1} k_{l}^{\prime}\left(x_{1}\right) / k_{l}\left(x_{1}\right)}\right] .
\end{aligned}
$$

In the situation where there is no salt inside the sphere (i.e., $\left.\kappa_{2}=0\right)$,

$$
D_{l}^{\mathrm{out}}(x, 0, \eta)=\frac{i_{l}(x)}{k_{l}(x)}\left[\frac{l \eta-x i_{l}^{\prime}(x) / i_{l}(x)}{l \eta-x k_{l}^{\prime}(x) / k_{l}(x)}\right] .
$$

Substituting these expressions into the series expansion for the Green's function yields the following:

$$
\begin{aligned}
G_{\mathcal{K}}\left(\mathbf{r}, \mathbf{r}^{\prime}\right)= & \frac{4 \pi \kappa_{1}}{\epsilon_{1}} \sum_{l m} i_{l}\left(\kappa_{1} r_{<}\right) k_{l}\left(\kappa_{1} r_{>}\right) \\
& \times\left[1-D_{l}^{\text {out }}\left(\kappa_{1} R, \kappa_{2} R, \eta\right) \frac{k_{l}\left(\kappa_{1} r_{<}\right)}{i_{l}\left(\kappa_{1} r_{<}\right)}\right] \\
& \times Y_{l m}(\theta, \phi) Y_{l m}^{*}\left(\theta^{\prime}, \phi^{\prime}\right)=\frac{e^{-\kappa_{1}\left|\mathbf{r}-\mathbf{r}^{\prime}\right|}}{\epsilon_{1}\left|\mathbf{r}-\mathbf{r}^{\prime}\right|} \\
& -\frac{4 \pi \kappa_{1}}{\epsilon_{1}} \sum_{l m} k_{l}\left(\kappa_{1} r_{<}\right) k_{l}\left(\kappa_{1} r_{>}\right) \\
& \times D_{l}^{\text {out }}\left(\kappa_{1} R, \kappa_{2} R, \eta\right) Y_{l m}(\theta, \phi) Y_{l m}^{*}\left(\theta^{\prime}, \phi^{\prime}\right) .
\end{aligned}
$$

The first term is the Green's function of a bulk electrolyte. The second term represents the influence of the dielectric particle. The corresponding expression for $\delta G_{\mathcal{K}}$ is

$$
\delta G_{\mathcal{K}}(\mathbf{r}, \mathbf{r})=-\frac{2 \kappa_{1}}{\epsilon_{1}} \sum_{l=0}^{\infty}(l+1 / 2) k_{l}^{2}\left(\kappa_{1} r\right) D_{l}^{\text {out }}\left(\kappa_{1} R, \kappa_{2} R, \eta\right) .
$$

\footnotetext{
${ }^{1}$ T. Arakawa and S. N. Timasheff, Biochemistry 21, 6545 (1982).

${ }^{2}$ C. Wagner, Phys. Z. 25, 474 (1924).

${ }^{3}$ L. Onsager and N. T. Samaras, J. Chem. Phys. 2, 528 (1934).

${ }^{4}$ D. Bratko, B. Jönsson, and H. Wennerström, Chem. Phys. Lett. 128, 449 (1986).

${ }^{5}$ R. Kjellander and S. Marčelja, Chem. Phys. Lett. 112, 49 (1984).

${ }^{6}$ R. Kjellander and S. Marcelja, J. Chem. Phys. 82, 2122 (1985).

${ }^{7}$ R. R. Netz, Phys. Rev. E 60, 3174 (1999).

${ }^{8}$ T. Croxton, D. A. McQuarrie, G. N. Patey, G. M. Torrie, and J. P. Valleau, Can. J. Chem. 59, 1998 (1981).

${ }^{9}$ G. M. Torrie, J. P. Valleau, and G. N. Patey, J. Chem. Phys. 76, 4615 (1982).

${ }^{10}$ G. M. Torrie, J. P. Valleau, and C. W. Outhwaite, J. Chem. Phys. 81, 6296 (1984).

${ }^{11}$ L. B. Bhuiyan, D. Bratko, and C. W. Outhwaite, J. Phys. Chem. 95, 336 (1991).

${ }^{12}$ P. Linse, J. Phys. Chem. 90, 6821 (1986).

${ }^{13}$ R. Messina, J. Chem. Phys. 117, 11062 (2002).

${ }^{14}$ J. Groenewold, J. Chem. Phys. 107, 9668 (1997).
} 
${ }^{15}$ A. L. Kholodenko and A. L. Beyerlein, Phys. Rev. A 34, 3309 (1986).

${ }^{16}$ R. D. Coalson and A. Duncan, J. Chem. Phys. 97, 5653 (1992).

${ }^{17}$ J. Ortner, Phys. Rev. E 59, 6312 (1999).

${ }^{18}$ R. R. Netz and H. Orland, Eur. Phys. J. E 1, 203 (2000).

${ }^{19}$ R. R. Netz, Eur. Phys. J. E 3, 131 (2000).

${ }^{20}$ R. R. Netz, Eur. Phys. J. E 5, 189 (2001).

${ }^{21}$ D. S. Dean and R. R. Horgan, Phys. Rev. E 69, 061603 (2004).

${ }^{22}$ A. M. Walsh and R. D. Coalson, J. Chem. Phys. 100, 1559 (1994).

${ }^{23}$ M. Boström, D. R. M. Williams, and B. W. Ninham, Langmuir 17, 4475 (2001).

${ }^{24}$ L. Lue, N. Zoeller, and D. Blankschtein, Langmuir 15, 3726 (1999).

${ }^{25}$ J.-P. Hansen and I. R. McDonald, Theory of Simple Liquids, 2nd ed. (Academic, London, 1986).

${ }^{26}$ J. D. Jackson, Classical Electrodynamics (Wiley, New York, 1975).

${ }^{27}$ R. L. Stratonovich, Dokl. Akad. Nauk SSSR 115, 1097 (1957).

${ }^{28}$ J. Hubbard, Phys. Rev. Lett. 3, 77 (1959).

${ }^{29}$ R. P. Feynman, Statistical Mechanics: A Set of Lectures (Addison-
Wesley, Redwood, CA, 1972).

${ }^{30}$ R. R. Netz and H. Orland, Eur. Phys. J. E 11, 310 (2003).

${ }^{31}$ H. Kleinert, Path Integrals in Quatum Mechanics, Statistics, and Polymer Physics, 2nd ed. (World Scientific, Singapore, 1995).

${ }^{32}$ J. L. Lebowitz and J. K. Percus, J. Math. Phys. 4, 116 (1963).

${ }^{33}$ Y. Levin, J. Chem. Phys. 113, 9722 (2000).

${ }^{34}$ M. Manciu and E. Ruckenstein, Adv. Colloid Interface Sci. 105, 63 (2003).

${ }^{35}$ J. M. Caillol, D. Levesque, and J. J. Weiss, J. Chem. Phys. 91, 5544 (1989).

${ }^{36}$ G. N. Watson, A Treatise on the Theory of Bessel Functions, 2nd ed. (Cambridge University Press, Cambridge, 1944).

${ }^{37}$ N. F. Carnahan and K. E. Starling, J. Chem. Phys. 51, 635 (1969).

${ }^{38}$ P. B. Petersen, R. J. Saykally, M. Mucha, and P. Jungwirth, J. Phys. Chem. B 109, 10915 (2005).

${ }^{39}$ G. Arfken, Mathematical Methods for Physicists, 3rd ed. (Academic, San Diego, 1985). 